and other developed countries, because effective treatments were available. As a result, the control group received alternative active treatments.

The FDA estimates that annually it receives data from around 575 foreign drug trials conducted without its knowledge, more and more of which come from trials run in the developing world. Currently, these trials must comply with the Declaration of Helsinki (or with local country laws, whichever offer the most protection) if sponsors want to use the data to win US marketing approval. The declaration, adopted in 1964 , and revised several times since, is today endorsed by medical associations from 85 countries. It is widely considered to be the bedrock of protection for research subjects. Its 1989 revision, which the FDA uses as its present standard, states that any patient in any trial "should be assured of the best proven diagnostic and therapeutic method".

Yet the FDA announced last month that it will shelve the declaration. Starting in October, the FDA intends to adopt a new standard it calls Good Clinical Practice (GCP), which is modelled on a 1996 document developed by drug regulators and pharmaceutical industry representatives from the United States, the European Union and Japan. Although GCP deals with subject protection, it is in essence a manual on how to conduct rigorous clinical trials, not a human-

rights document. For instance, whereas Helsinki explicitly discourages the use of placebos for serious conditions where proven therapies exist, GCP is silent on this issue. So under the GCP guidelines, the FDA could accept data from Surfaxin placebo trials of the future.

The FDA argues that it should not be bound by Helsinki because the declaration is devised by a group it does not control, and is subject to periodic revisions that could confuse trial sponsors or contradict US law. But it is tempting to conclude that the FDA is drop-

"The FDA risks sending a message that ethical considerations are expendable when research subjects live half a world away."

ping Helsinki not because it is changeable, but because the agency disagrees with the way it has been changing - in particular with its constraints on the use of placebos. (The US agency is more favourably disposed to placebo use than, say, its European counterparts.)

It makes sense for the FDA to adopt the GCP standard, giving foreign-based researchers guidelines that should help them generate the best data. But if the FDA jettisons Helsinki, the critical underpinning for such efforts, it risks sending a message that ethical considerations are expendable when research subjects live half a world away.

\section{The Universe at home}

\section{The digitization of astronomy is a transformation and a delight for both amateurs and professionals.}

T o look through even a small telescope at the greatness of the sky is a heady thing. It is not just the aesthetic delight of stars like grains of sand, or cloud-decked galaxies like tiny hurricanes in seas unseen; nor is it merely the knowledge of immensity that comes with understanding that each grain is a sun bright and ancient, each cloud an unknowable plurality of worlds. It is the sheer cosmic kick that comes from having the rods and cones of your retina stirred by photons that have been travelling for so long that mountains once young have crumbled to the sea. A ray of light that begins in another galaxy and ends in your nervous system is a miracle never to be tired of.

This continuing appeal of amateur astronomy should, light pollution permitting, see children and pensioners in their back gardens and up their local hills for as long as there are telescope makers to satisfy them. Online services such as Google Sky and Microsoft's new WorldWide Telescope allow users to scan the sky at higher resolution and in more wavelengths than amateurs could ever do, yet there is no reason to fear that they will bring those skywatchers indoors for good. Quite the reverse: their on-screen wonders feed the appetite to see for yourself.

Better still, the Internet allows the aggregation of observing time - both for those with telescopes and without. Amateurs following up newly discovered asteroids get the orbital elements from the website of the International Astronomical Union's Minor Planet Center. Galaxy Zoo, a site where a million galaxies await classification, puts the profusion of amateur eyeballs to further use, and has produced not only an unexpected level of interest but also some sound publications. Harnessing the pattern-recognition skills of people around the world who have no astronomical equipment other than a broadband connection may permit a range of similar projects in the future, as new surveys produce images at ever greater rates.

The Astrometry.net software we report this week (see page 437) offers new ways for the Internet to combine the observations of amateurs and professionals, past and present. It aims to provide the correct spatial and temporal coordinates for any picture of the sky submitted to it, be it a recent CCD file or a glass plate found in GreatAunt Herschel's attic. Its creators hope, with suitably astronomical ambition, to identify, and possibly assimilate, every image of the sky ever made. In doing so, they imagine discovering new truths about the way the sky changes over time - to recognize the transient, the unexpected, the hitherto unnoticed but nevertheless captured.

This is the sort of totalizing impulse that normally deserves scepticism, if not disquiet. In this case, though, it is hard not to see it as noble. The idea that all the solitary skywatchers are engaged in a single study, linked by ties of knowledge even as they stare upwards on their own, has always had its poetic truth. To make it practically true is a fine aspiration. Walt Whitman's poetic narrator may have

Wander'd off by myself, In the mystical moist night-air, and from time to time, Look'd up in perfect silence at the stars

- but there is no need to reject the learned astronomers' proofs and figures, charts and diagrams in order to experience that which enriches the soul. Observers of all sorts will soon be able to add to the glories of the endlessly interconnected inner world of the Internet while losing nothing of that precious and primal communion with the cosmos. 